Voix et Images

voixetimages

\title{
Du féminin encore et encore
}

\section{Claudine Potvin}

Volume 20, numéro 3 (60), printemps 1995

André Brochu

URI : https://id.erudit.org/iderudit/201199ar

DOI : https://doi.org/10.7202/201199ar

Aller au sommaire du numéro

Éditeur(s)

Université du Québec à Montréal

\section{ISSN}

0318-9201 (imprimé)

1705-933X (numérique)

Découvrir la revue

Citer cet article

Potvin, C. (1995). Du féminin encore et encore. Voix et Images, 20(3), 702-709.

https://doi.org/10.7202/201199ar d'utilisation que vous pouvez consulter en ligne.

https://apropos.erudit.org/fr/usagers/politique-dutilisation/ 


\section{Du féminin encore et encore}

\section{Claudine Potvin, Université d'Alberta}

Suite à une première anthologie parue en 1992, qui répondait alors à un désir de reprendre dans une perspective chronologique quelques réflexions critiques sur les classiques québécois de l'écriture au féminin, Lori Saint-Martin a réuni dans son deuxième volume de L'Autre Lecture. La critique au féminin et les textes québécois ${ }^{1}$ une série d'essais centrés sur la production littéraire féminine des années soixante-dix et quatre-vingt. On retrouvera donc ici quatorze articles qui ont d'abord été publiés dans les années quatre-vingt (Féral, 
Godard, Lamy, Forsyth, Moss, Smart) et quatre-vingt-dix (Gauvin, von Flotow, Lotbinière-Harwood, Gould, Lequin, Dupré, Saint-Martin). Si, dans un premier temps, cet ouvrage offre un regard diachronique sur un passé relativement proche, les années d'affirmation du féminisme, suivi d'un questionnement de la période contemporaine à travers des textes plus récents, la structure tripartite du livre (théorie, analyse et critique ou exploration) propose de repenser la problématique de l'écriture des femmes en synchronie.

L'utilité de L'Autre Lecture, titre que l'éditrice emprunte à l'article bien connu de Suzanne Lamy qui ouvre la collection, ne fait aucun doute. Le fait de rassembler des textes critiques éparpillés permet de donner une vue d'ensemble nécessaire et de fournir un outil de travail important aux chercheuses et aux chercheurs du monde académique. Comme le signale Lori Saint-Martin, et comme l'a montré un symposium sur l'écriture au féminin 'et l'institution littéraire que j'ai organisé en 1989 , les femmes se parlent le plus souvent entre elles, augmentant de la sorte un effet de ghettoïcisation entretenu par les appareils institutionnels: numéros de revues qu'elles seules lisent, colloques auxquels ne se pointent que quelques éléments masculins, cours de littérature et programmes d'études qui ne paraissent s'adresser qu'à des étudiantes. Il semble bien, pour reprendre Suzanne Lamy, que les textes de femmes n'appartiennent pas au grand tout de la littérature. L'écoute demeure trop souvent obligée ou polie. Une collection comme celle-ci vient nous rappeler que la lecture au féminin est toujours aussi nécessaire.

De prime abord, on pourrait s'interroger justement sur la pertinence de rassembler ces travaux puisque dans les deux premières parties du volume, à l'exception de deux collaborations, celles de Lotbinière-Harwood et de Gould, les articles retenus datent de la première moitié des années quatre-vingt et se penchent essentiellement sur une période qu'on dit révolue, celle des prises de parole enflammées des Brossard, Théoret, Bersianik, Marchessault, dont on a beaucoup discouru. À mon avis, c'est précisément parce qu'il y a eu déplacement et non effacement qu'il importe plus que jamais de réunir et relire ces textes. En ce sens, l'intérêt de cette collection est que si elle réaffirme un grand nombre de préceptes des premières bibles littéraires féministes, elle interroge parallèlement, plus spécifiquement dans la troisième partie, les définitions, les classifications, les genres, sa propre lecture, et ce à partir d'un lieu éclaté, celui du début des années quatre-vingt-dix. De post-féminisme on ne peut guère parler toutefois; que le sujet féminin se positionne différemment ne signifie pas la garantie de son autonomie ni son inscription définitive dans 
l'ordre du symbolique. Le post-féminisme représente avant tout le regard d'un-e autre qui se situe déjà après les événements mais sans y avoir participé et qui ne retient que le grand dérangement d'une époque soi-disant terminée.

Or, ce livre nous permet une nouvelle incursion dans un des champs culturel, politique et littéraire de la littérature québécoise par le détour de ce qu'on nomme la critique au féminin, donc en fonction d'un angle marqué, comme on le dit du genre. Mais tout angle suppose une "marque " que la critique dite "universelle" ne définit pas ou peu dans son discours. Faut-il ajouter que, homme ou femme, on n'écrira plus de la même manière au Québec après l'émergence de la parole féminine, que l'on se place à l'intérieur ou en marge. d'un mouvement qui cherchait à transformer la pensée à travers le langage. Cependant, la critique continue bien souvent à ignorer ou à bouder ce qui se brasse du côté pratique et théorique du féminin. Si la postmodernité et le féminisme se rejoignent dans leur déconstruction des métarécits et des discours fondés sur la raison, la vérité, le progrès, l'unicité comme le remarque Gould à propos du Désert Mauve de Brossard, les pratiques féministes ne s'en trouvent pas moins évacuées au nom de cette même parenté. Ainsi, tout un rapport à la différenciation sexuelle (gender), au corps, au quotidien, au détail, à une forme de circulation ou un mode d'habiter l'espace, à l'intime, à l'affect et à l'érotique, au moi, à la différence, se trouve à nouveau escamoté.

Les études de la première partie de L'Autre Lecture reprennent précisément ces notions devenues des clichés au fil des ans, à savoir que le discours théorique véhiculé par nombre de méthodes d'approche critique (structuralisme, marxisme, sociocritique, psychanalyse, sémiotique) ne peut se développer en dehors de la constitution d'un sujet féminin, c'est-à-dire sexuel. Pour Suzanne Lamy, l'écriture-femme, bien que pas toujours de façon évidente, a partie liée avec la délivrance des consciences, la représentation d'un non-vu et d'un non-dit. À la question "qu'est-ce qu'écrire au féminin ", elle répondait jadis que "Les affrontements du réel et de l'imaginaire, l'émergence d'une nouvelle identité pour la femme, les investissements qui orientent et colorent le texte, en conditionnent la structure, les rythmes, la rhétorique, font qu'il y a écriture et au féminin. (p. 25). Lamy réclamait d'un côté une lecture au féminin rigoureuse et de l'autre, une ouverture de la part des lecteurs accrochés à un concept critique d'objectivité et d'universalité foncièrement restrictif, la norme passant toujours par le critique mâle blanc, lettré, phallocrate, hétérosexuel, petit bourgeois. Comme le déclare celle qui a fortement 
influencé l'articulation de tout un discours critique, la lecture au féminin est un outil épistémologique qui vaut tous les autres en ce qu'elle peut elle aussi produire du sens, ce qui est encore vrai à l'heure actuelle. L'invitation lancée aux compagnons lecteurs par Lamy se précise et se justifie dans son deuxième article, "Des enfants uniques, nés de père et de mère inconnus ", dans lequel l'auteure affirme la dissidence des textes de femmes: rupture face aux formes traditionnelles, brouillage des conventions littéraires, éclatement du langage, multiplicité, pluralité, polytonalité, intertextualité vue comme un réseau de connivences, traversées du corps et de l'imaginaire, complicité avec le monde et la culture des femmes, amorce d'une civilisation où le collectif ne barre plus l'individuel.

Les articles de Josette Féral et de Louise Forsyth vont dans le même sens. La première insiste davantage sur le concept de gender tel qu'on le retrouve chez un grand nombre de théoriciennes féministes américaines (Jardine, Showalter, De Lauretis, Suleiman) alors que la seconde s'attarde sur le rôle de la critique en général et sur les problèmes qui se posent à la recherche et à la critique féministes en particulier. Selon Féral, l'origine de toute parole se situe là où se joue la sexuation du discours, d'où la nécessité de repenser l'écriture au féminin non pas en termes de non-identité face à l'Autre ou de manque, mais à partir d'un questionnement de la théorie qui évacue la femme. Par conséquent, les instruments critiques (Féral examine l'exemple de la sémiotique et du structuralisme qu'elle jumelle et celui de la psychanalyse) qui ne tiennent pas compte de la différence sexuelle et du contexte de production ne paraissent pas véritablement aptes à parler de cette nouvelle réalité féminine. Bref, Féral suggère de considérer les présupposés philosophiques à l'origine de toute réflexion d'ordre critique et conclut que tout discours critique au féminin implique un processus de création en ce qu'il se transforme en un nouveau texte, une nouvelle fiction ajouterais-je, elle-même objet de lecture. Forsyth présente également la critique féministe comme une démarche créatrice qui remet en cause les valeurs et le langage de l'institution littéraire patriarcale qui ont bloqué l'appréciation des œuvres de femmes. Le triple défi consiste donc à établir une complicité au niveau de la réception du texte, à saisir la position théorique appropriée pour en dégager la force et à refuser de traiter avec ceux qui nient le dialogue. Les lectures au féminin mettent ainsi en place des stratégies de résistance génératrices d'énergies propres à assurer la reconnaissance et la survie d'une culture au féminin.

L'exemple d'une pratique de la traduction pensée comme une réécriture au féminin, telle qu'exercée par Susanne de Lotbinière- 
Harwood, s'avère certes révélateur dans ce contexte. À qui appartient le langage? D'où parlent-ils? D'où parlent-elles?, s'interroge cette dernière, pour qui "Toutes les femmes sont bilingues" (p. 59) puisque forcées depuis toujours de traduire la langue dominante en leurs mots. Or, tout acte de lecture étant codé d'avance en fonction d'une grammaire répressive et exclusive, la position de la femme traductrice se doit d'être subversive. "Le féminisme, écrit Lotbinière-Harwood, amène à pratiquer la traduction comme prise de parole politique et à revendiquer sa reconnaissance comme activité créatrice légitime ayant une signature dont le travail n'est pas entièrement soumis à l'auteur-ité de l'œuvre d'origine" (p. 64). Repenser le corps traduisant en termes de performance débouche sur le mouvement qu'autorise l'écriture au féminin et sur un travail de collaboration entre co-créatrices, sur une conversation infinie. Les vocables "re-belle" et "infidèle" utilisés par Lotbinière-Harwood pour décrire son travail proposent, sur le mode de la rébellion et de l'infidélité au texte original, de déconstruire le modèle d'une parole et d'un sens premiers, idéaux, absolus, refusant une fois pour toutes la copie, la reproduction du même ad infinitum et ad nauseum.

Ces considérations initiales se trouvent renforcées dans la deuxième partie de l'anthologie par les propos de Barbara Godard sur la poétique féministe manifeste dans les numéros que La (Nouvelle) Barre du jour a consacrés à l'écriture au féminin. Par ailleurs, les trois lectures de Jane Moss, Patricia Smart et Karen Gould illustrent également et à plus d'un titre ces commentaires théoriques. La critique au féminin s'est considérablement développée au cours des dernières années et la production littéraire des femmes n'a cessé d'augmenter. Il n'est donc pas facile d'opérer une sélection et en ce sens tout choix sera nécessairement discutable. Les articles retenus par Lori SaintMartin semblent l'avoir été en fonction d'un désir d'illustrer ou de compléter les réflexions théoriques du début, de la problématique traitée et de l'autorité des critiques dans le domaine ainsi que de la reconnaissance des auteures par l'institution littéraire. Portant tantôt sur le théâtre de Jovette Marchessault, tantôt sur le réalisme de France Théoret ou l'esthétique féministe et postmoderne de Nicole Brossard, ces analyses textuelles mettent en évidence les préoccupations et les marques de l'écriture au féminin répertoriées par Godard. Dans tous les cas, il est question de transgression langagière, de subversion des mythes, du rejet de la tradition et de toute vision totalisante du savoir, de l'écriture comme recherche et exploration, de naissance à soi et au monde, du désir de dire l'expérience, le quotidien, de transformation du réel, de reconstruction de la culture, d'une relecture de l'Histoire, 
de l'émergence du sujet féminin et de son insertion dans le social et le politique, de pluralité des voix.

C'est bien autour de la notion de pluralité que le troisième volet de cette collection s'articule; les critiques s'y intéressent tout particulièrement aux manifestations plus récentes de l'écriture des femmes. Le sous-titre "Genres, mouvements, tendances" annonce un mélange: référence à la mixité de la production sans doute, allusion au décentrement, aux bifurcations et aux multiples voies/ voix empruntées par les écrivaines, enfin déplacements textuels auxquels la critique fait écho. Les lectrices et lecteurs trouveront ici cinq études sur l'essai au féminin, l'érotisme hétérosexuel, l'exil et l'écriture migrante, le discours poétique et le métaféminisme dans la nouvelle prose féminine. À part l'article de Lise Gaùvin qui fait le constat de la marginalisation du discours référentiel féminin et qui pose la complexité de la position du je de l'essayiste femme, le paradigme dominant de ces écrits repose sur l'observation d'une certaine déviation textuelle et idéologique dans la fiction des années quatre-vingt face à l'écriture des pionnières et face au dogme féministe. Les auteures étudiées par Louise von Flotow, Lucie Lequin, Louise Dupré et Lori Saint-Martin, poètes, romancières ou nouvellistes, ne s'inscrivent apparemment plus dans les normes: retour au récit linéaire et à la lisibilité chez certaines, prépondérance de la passion amoureuse et de l'intime chez d'autres, essoufflement face au mouvement féministe et atténuement de l'esprit de révolte chez toutes. Ainsi, la quête d'une écriture spécifiquement féminine semble abandonnée au profit d'une omniprésence de l'expérience personnelle.

Néanmoins, ces lectures critiques révèlent au contraire que la théorie féministe travaille la narrativité et que si on ne peut plus parler d'écriture "manifestaire", la parole des femmes s'inscrit toujours sur fond de rébellion. Ces essais tendent à établir qu'il n'y a pas régression à la langue d'avant le mouvement des femmes ni dépassement du féminisme. Louise Dupré invoque la multiplicité des postures. Les voix migrantes auxquelles se réfère Lucie Lequin témoignent d'une écriture variée, ouverte, plurivoque. La traversée des frontières, entre la réalité et l'imaginaire, entre la parole et l'action, s'accommoderait d'ailleurs assez mal d'un discours unique, monolithique, l'écriture migrante se situant davantage du côté de la dérive et représentant avant tout l'expression d'un acte de transmutation et de création d'une nouvelle identité, d'un nouveau territoire. Toute écrivaine s'engage dans un processus de migration au moment où elle prend la plume. Selon Lori Saint-Martin, les textes d'auteures telles que Suzanne Jacob, Francine 
Noël, Monique La Rue, Lise Harou par exemble, témoignent d'une évolution et d'une transformation par rapport à la textualité des années soixante-dix. Saint-Martin qualifie très justement ces écrits de métaféministes entendant par ce terme une écriture qui remet en cause "les stratégies et les orientations des aînées féministes tout en intégrant à la fiction des préoccupations similaires"(p. 164). Elle ajoute encore que ces textes "n'évacuent pas le féminin, mais l'absorbent, l'interrogent, le font évoluer" (p. 169) et ouvrent ainsi de nouvelles perspectives sur les femmes. Par ailleurs, ces textes obligent la critique au féminin à repenser son approche, à redéfinir constamment ses assises et à renouveler son discours critique. Toute forme d'essentialisme ne sera plus jamais possible; on ne peut plus faire allusion à "la femme" ni à son désir ni à sa parole sans spécifier de quel lieu on/elle parle. L'étude de Louise von Flotow sur l'érotique dans les récits brefs d'Anne Dandurand le montre bien. En effet, comment se placer entre l'érotique et le pornographique, la violence et l'amour, la représentation du corps féminin et le déplacement/ éclatement du corps masculin? Cette dernière partie de L'Autre Lecture, pour moi la plus intéressante parce qu'elle suggère de nouvelles pistes, indique que la critique au féminin bouge au rythme des textes auxquels elle se voit confrontée.

En conclusion, malgré un certain effet de dispersion inévitable dans ce genre d'ouvrage et l'inégalité des contributions, la thématique générale assure l'unité de l'anthologie. Le livre de Lori Saint-Martin a l'avantage d'offrir à la fois un rappel théorique et une projection critique. En effet, l'ensemble des problématiques discutées donne un aperçu synthétique des démarches féministes critiques des dernières années. Parallèlement, cette collection permet de saisir, à travers une lecture critique rigoureuse, le mouvement de l'écriture au féminin au Québec au cours des deux décennies antérieures. Évidemment, notre perception demeure superficielle puisque le découpage est nécessairement arbitraire. À part l'introduction dans laquelle Lori Saint-Martin résume sommairement le contenu, ces études ne s'accompagnent pas d'une analyse contextuelle et théorique de l'ensemble. Là n'est pas la fonction d'une anthologie de la sorte; il s'agit plutôt de mettre en circulation un document qui permette aux lectrices et lecteurs de retrouver sous un même couvert un bon nombre d'études importantes sur le sujet. D'où la pertinence d'une publication comme celle-ci pour celles et ceux qui travaillent dans ce domaine, qui enseignent la littérature des femmes ou la littérature tout court, mais aussi pour toutes les lectrices et tous les lecteurs sensibles à la mise en place et au fonctionnement d'une écriture, voire de l'écriture. 
1. Lori Saint-Martin, L'Autre Lecture. La critique au féminin et les textes québécois, tome II, Montréal, XYZ éditeur, coll. a Documents ", 1994, 194 p. 